\title{
Use of the Flash Technique in EMDR Therapy: Four Case Examples
}

\author{
Philip Manfield \\ John F. Kennedy University, Berkeley, California \\ Joan Lovett \\ Healthy Perspectives, Berkeley, California \\ Lewis Engel \\ San Francisco, California \\ David Manfield \\ EMDR Associates, Lake Oswego, Oregon
}

\begin{abstract}
This article introduces the flash technique, a new technique used during the preparation phase of eye movement desensitization and reprocessing (EMDR) therapy to facilitate processing of intense, traumatic memories that clients might otherwise be resistant to access. Anecdotal evidence suggests that this technique may make it possible for clients to access these memories initially in a minimally disturbing way, reducing their emotional intensity so that they can then be more easily and fully processed using EMDR therapy. The technique appears to be easily tolerated by clients of all ages, including children; and to be rapid and relatively painless for clients, even those with particularly disturbing target memories; and can be easily taught to clinicians. It has the distinct feature that clients who are avoiding a terribly disturbing memory can be offered a way of processing it without having to bring it clearly to mind. Four case examples, in which the technique was used by four different clinicians, are presented briefly. Suggestions are made for further study. This article hypothesizes various mechanisms of action and discusses the effects in terms of memory reconsolidation theory.
\end{abstract}

Keywords: flash technique; eye movement desensitization and reprocessing (EMDR); memory reconsolidation; subliminal; working memory; trauma

$\mathbf{E}$ ye movement desensitization and reprocessing (EMDR) is a treatment developed and introduced by Francine Shapiro (1989) originally for the treatment of clients who have experienced single-incident traumas that resulted in posttraumatic stress disorder (PTSD). EMDR therapy (Shapiro, 2001) is now recognized as an effective method of treatment for a wide range of trauma-related psychological conditions. During EMDR therapy, the client focuses on the disturbing incident while performing a presenttime, dual attention task, most commonly rapid eye movements (EM) guided by the bilateral stimulation (BLS) of visually following the therapist's moving finger. The EMDR protocol involves eight phases: client history, preparation, assessment, desensitization, installation, body scan, closure, and reevaluation.

\section{The Eight Phases of EMDR}

\section{First Four Phases}

This article is concerned with the first four phases of EMDR. The first two, client history and preparation, enable the therapist to formulate the case, prepare the client for EMDR processing, and select an appropriate target memory (target) to be processed. The third phase, assessment, is intended to determine the components of the memory and to establish baseline measures by which progress in processing can be evaluated. The subjective units of disturbance (SUD; Wolpe, 1958) scale is used to evaluate the client's level of disturbance. During the fourth phase, desensitization, the memory is processed with the help of BLS and, sometimes, cadence 
comments (P. Manfield, 2013; described in Shapiro, 2001, p. 175), brief evenly spaced comments without much particular meaning, such as "Just notice," "That's right," and "Good."

\section{Last Four Phases}

In successful EMDR processing, the next three phases occur after a memory has been fully desensitized. Installation strengthens the adaptive adult perspective that has presumably replaced the cognitive distortion originally associated with the memory. The body scan checks for residual disturbing sensations related to the memory. Closure prepares the client to leave the session in a state of emotional equilibrium and provides instructions for the time between the current and next session. The eighth phase, reevaluation, takes place during the following session when the therapist checks to see how the processing results have endured, and if further processing is necessary for that memory.

\section{Addressing Intense Trauma}

\section{Processing Overwhelming Trauma}

There are some clients whose traumatic material is too disturbing for them to remain emotionally stable when accessing it, and they tend to become overwhelmed or shut down during treatment. Several therapies include elements that allow clients to process overwhelming trauma with a more moderate level of activation. Some, including BLS in EMDR, the counting method (Ochberg, 1996), and progressive counting (Greenwald, 2008), appear to create a task that taxes working memory (WM) so that clients are unable to simultaneously do the task and maintain a vivid and disturbing memory of the trauma (Maxfield, Melnyk, \& Hayman, 2008; Shapiro, 2001).

In EMDR therapy, various techniques has been developed to help those clients whose treatment engagement is impaired by over- or undermodulation. In addition to extensive preparation, some procedures are conducted when desensitization stalls. Some techniques entail graduated exposure or substituting a modified, less disturbing memory for the original memory (Forgash, 2004; Gomez, 2014; Shapiro, 2001). Some limit the time that clients think about the memory to as little as 2 seconds and then gradually increase that amount of time (Greenwald, 2008; Kinowski, 2003; Knipe, 2008). Others strengthen clients' connections to their positive adult identities during processing so that traumatic material can be viewed from a distance rather than reexperienced
(Forgash, 2004; Korn \& Leeds, 2002; U. Lanius, 2005; Leutner \& Cronauer, 2010; P. Manfield, 2010; P. Manfield \& Shapiro, 2003; Shapiro, 2001). Of relevance in this article are the techniques of paired titration (Kinowski, 2003) and the observer position (Shapiro, 2001).

\section{Paired Titration}

Kinowski (2003) adapted Levine's (1997) somatic experiencing pendulation technique to EMDR in her paired titration process. She first helped the client develop a connection with a resource image that made the client feel more resilient and then instructed the client to limit his or her exposure to the traumatic material by telling him or her to go just to the edge of the trauma. In addition, she limited the amount of time the client was exposed to the memory, doing this by guiding the client verbally in real time through the steps of connecting to the resource, going to the edge of the trauma, and finally returning to the resource. In her protocol, clients repeatedly alternate between the resource and the traumatic material. Over time, Kinowski made the exposures progressively longer and more central to the trauma. Throughout the process, she evaluated if each contact with the trauma had been too intense by whether the client reported difficulty returning to the resourced state.

\section{Maintaining the Observer Position}

Shapiro (2001) stated that "the components [of EMDR] . . are geared to convince the client that she is larger than the pathology and can effectively remain an observer of its previously overwhelming effects" (p. 141). The observer role (present orientation) seems to prevent reliving the painful trauma experience. Shapiro posits that the observer stance enhances mindful awareness.

\section{Related Research}

\section{Memory Reconsolidation Research}

Memory reconsolidation is a term to describe how memories that have been retrieved from long-term memory into working memory are again stored in long-term memory. It is an important process because it can explain how altered memories can permanently replace the originals, allowing disturbing memories to become benign. This permanent transformation process is referred to by the somewhat confusing term erasure (Elsey \& Kindt, 2017). Actually, only the traumatic quality of the memory is "erased"; the narrative of the event remains intact. 
Memory reconsolidation research provides some understanding of the conditions necessary for erasure to occur. Studies suggest that erasure of conditioned fear responses in various animal species only occurs when the animal accesses the original memory that caused the fear response and there is also a prediction error (Dudai, 2004; Pedreira, Pérez-Cuesta, \& Maldonado, 2004). In other words, when the animal encounters a stimulus like the one that was present in the original emotionally linked memory, it retrieves the original memory from long-term memory into WM. Then, for erasure to occur the animal must encounter new information that presumably necessitates an update of the original memory, to make it more accurate. Researchers have identified a period of up to 6 hours in which the memory in WM becomes labile and updating can occur (Elsey \& Kindt, 2017).

Ecker, Ticic, and Hulley (2012); Hupbach, Gomez, Hardt, and Nadel (2007); Lee (2009); Sevenster, Beckers, and Kindt (2013); and others offer evidence to suggest that memory erasure in humans also requires both retrieval and a prediction error. The prediction error (e.g., "I'm safe now" contradicts the initial prediction, "I will be harmed") is incorporated into the memory that has been retrieved, and the altered memory can then replace the original memory when it is stored back (reconsolidated) into long-term memory. Memory reconsolidation theory suggests that this alteration of the original memory, once reconsolidated, is permanent.

\section{EMDR Can Permanently Alter Traumatic Memories}

The observable impact of successful EMDR suggests that it accomplishes permanent change (erasure) and therefore must meet both of these required conditions. EMDR involves the full accessing of the traumatic memory during the assessment and desensitization phases of EMDR treatment, and it includes an experience for the client that is contradictory to what was expected. During EMDR, the experience of recalling the memory with simultaneous BLS, along with the absence of reexperiencing of overwhelming affect or intense sense of danger or shame probably constitutes the required prediction error.

\section{Necessary Characteristics of Memory Retrieval}

The research on memory reconsolidation has shown that the memory must be retrieved for erasure to occur, but it is vague about what constitutes the necessary retrieval. In EMDR therapy, for instance, memory retrieval is explicit, overt, and usually emotional.
The assessment phase encourages memory retrieval when the client brings up visual, cognitive, emotional, and somatic aspects of the target memory, all of which, along with BLS, contribute to the client's retrieval of the memory. In fact, most experienced EMDR clinicians will attest that when clients avoid fully accessing a target memory during EMDR therapy, progress is often halted.

Is it necessary, however, for memory retrieval to include such a conscious and emotional connection to the memory. At least one animal study concludes that reconsolidation and erasure can be accomplished even if the affective recall ("expression") of the trauma memory is chemically blocked (Barreiro, Suárez, Lynch, Molina, \& Delorenzi, 2013). This appears to be the case as well with the flash technique (FT) described in the following text.

What is the minimum length of time that the memory must be held in WM to satisfy the retrieval requirement? There is a large body of research demonstrating that as little as 4 milliseconds, four thousandths of a second, of access to a visual stimulus is sufficient to stimulate full information retrieval and processing of the stimulus (D. Manfield, 1986; Silverman \& Weinberger, 1985; see Mansfield, 1997, for an extensive discussion of relevant research). Furthermore, a visual stimulus of less than 37 milliseconds is unlikely to be consciously perceived or understood but, nevertheless, will have a demonstratively greater psychological impact than stimulation that is consciously recognized, which in fact does not have any significant impact (Mansfield, 1997). Most of this research has been done with subliminal, visual, word messages, both positive and negative, but some have involved subliminal exposure to visual images as stimuli (Meyer \& Waller, 1999). Although all the subliminal messaging research focused on perceived external stimuli, it is reasonable to suspect that similar time thresholds would apply to stimuli that originate in long-term memory.

What is significant about this research for this article is that (a) at least in animals, it is possible that trauma memories can be retrieved without their emotional component, and still be altered, and reconsolidated; (b) an image or message can be accessed fully enough to significantly impact the viewer, even if the exposure was so brief as to leave the viewer with no recall of what was seen; and (c) images or messages that appeared long enough to be consciously recognized actually had no observable effect on the viewer. Based on these research outcomes and our experience with FT we suspect that the extremely brief exposure to the trauma memory during FT may be 
sufficient to meet the retrieval requirement proposed by Ecker et al. (2012), Lee (2009), and others.

\section{The Flash Technique}

FT is employed during the preparation phase of EMDR as a rapid and relatively painless way of reducing the intensity of extremely disturbing memories so that they can be easily processed in the remaining phases of standard EMDR treatment. It is recommended for clients who are highly apprehensive of accessing their traumatic material, who dissociate when accessing the memory, who become emotionally overwhelmed, or who are resistant to visiting the memory. It can also be used in place of lengthier preparation activities for some clients. The goal of the intervention is to painlessly reduce the disturbance associated with a target memory to a level that the client is no longer resistant to fully accessing it and processing it with standard EMDR. Clients are advised that this can be a way that they can reduce memory-related distress without having to think about it. If they respond with pronounced skepticism, the therapist suggests that the procedure may not work but that the client has nothing to lose except maybe 10 minutes. The intervention may take from 10 to 45 minutes (Table 1 ).

Before a memory becomes significantly activated, the client is asked to start from a resourced or relatively neutral state and, when ready, to flash on the memory for a small fraction of a second and then let the therapist know when he or she has returned to the neutral or resourced state. The resourced state can be generated by any thought, memory, or image that gives the client a sense of well-being or calmness. Before and during the flash, BLS is used to guide the client in making slow EM. (i.e., approximately 2-3 seconds for each left-right-left pass). The client is asked to indicate when he or she has returned to the neutral or resourced state. The full set is usually four to five passes with the client accessing the memory in a flash after the third or fourth pass; however, some clients initially may take longer.

\section{Keeping the Flash Brief}

The client is cautioned not to think about the memory or rehearse before the flash, but, when ready, simply to think of the memory or the disturbance for a tiny fraction of a second, and then come back to the neutral or resourced state. The flash can be described as a flicker or a blur. Clients can be told that the most common mistake clients make in flashing is to access the memory for too long because that might make the flash more disturbing than desired. They can be reassured that it is all right if they flash so rapidly that they are not sure if they actually connected to the memory and that in fact this is often when the most rapid effect is achieved. A metaphor that works well is to equate the flash to passing a finger through a candle flame. If it is done quickly, it should not involve pain.

\section{Subliminal Stimuli}

It is possible that when a client is pushed to access a memory so fast that it is barely a blur, there is an effect similar to that identified in the subliminal messaging research just discussed (Mansfield, 1997). This research found that if the stimulus remained visible long enough for the client to consciously recognize it, no psychological effect was observed. To have an effect, the message needed to be brief enough so that the subject could not think about it consciously and defend against it. Similarly, FT appears to work best when the flash is so rapid that the client does not have time to think about or vividly recall the memory.

As in paired titration, clients are asked whether they had difficulty coming back to the neutral state after each flash. If so, they are asked to make the next flash considerably briefer. After a set, the therapist does not ask about the SUD levels or "What came up?"

TABLE 1. Summary of the Treatment of Four Traumatic Events

\begin{tabular}{ccccc}
\hline Case & Pre-FT SUD & Post-FT SUD & PTSD & Minutes of FT + EMDR \\
\hline 1 & 10 & 0 & Yes & 20,45 \\
2 & 10 & 0 & Yes & 10 \\
3 & 9 & 0 & Yes & 45 \\
4 & 10 & 0 & Yes & 45 \\
\hline
\end{tabular}

Note. FT $=$ the flash technique; SUD $=$ subjective units of disturbance; PTSD $=$ posttraumatic stress disorder; minutes of FT + $\mathrm{EMDR}=$ time spent processing one particular memory. 
because these questions can encourage clients to think of the disturbance, which defeats the intent of FT. Once clients can flash and return to the neutral state without difficulty multiple times, the therapist can instruct them to flash three times during each set. After about three of these triple flashes, clients are asked if they notice any difference in the level of disturbance evoked by the target memory. If clients report significantly lower disturbance or difficulty accessing the memory, the therapist can ask the SUD level associated with the memory. Proceeding to the assessment phase of EMDR once the initial disturbance has been reduced allows clients to process the remaining disturbance, some of which may be associated with secondary channels of the target memory, and some of which may be related to cognitive distortions or body sensations associated with the trauma memory.

\section{Abreactions Are Rare}

Typically, clients do not become activated during FT processing. On rare occasions during an initial flash, a client connects with the memory much longer than instructed and begins to abreact. To date, such abreactions have been mild because these clients were still not accessing the full strength of the memory, and the emotional intensity was dissipated during one or two full sets of rapid EM with cadence comments. Among other things, the rapid EM with cadence comments seem to help the client contain affect as they appear to do during EMDR Phase 4.

\section{Flash Technique Starts From a Neutral or Resourced State}

Optimally, FT starts from a neutral or resourced state during the preparation phase of EMDR therapy. Initially, for especially disturbing memories, the therapist can help the client develop a safe or calm place to focus on before FT. The client is asked specifically not to describe or even think about the target memory before flashing and not to rehearse or think about what he or she is going to think of during the flash. This is done to keep the client's fear of overwhelming affect from being stimulated because once that fear has become strong in these clients, avoidance or dissociation is more likely.

If the client is somewhat activated at the outset, he or she can be instructed to think of the safe, calm, or pleasurable state and then allow the slow EM (Kreyer, \& Egon, 2008; Schubert, Lee, \& Drummond, 2011) to help her relax further before attempting to flash. Most clients seem to be able to accomplish this.

\section{Speed of Eye Movements}

During FT, EM are slow, approximately $2-3$ seconds per pass. This speed is too slow to tax WM (van Veen et al., 2015), so it does not seem to impede the brief accessing of the trauma memory the client does during FT. As will be explained later, taxing WM with faster EM does not appear desirable for FT. The slow EM do seem to help the client to relax into a neutral or resourced state before flashing (Kreyer \& Egon, 2008), which is useful for FT, and it seems to provide a structure that appears helpful for many clients.

\section{Clients Are Reassured With the Description of Flash Technique}

To help clients understand that FT is designed to keep them from becoming overwhelmed, therapists can tell them that their contact with the disturbance will be so brief that there will be no time for them to (a) become upset, (b) bring up a well-formed image, or (c) to have unpleasant thoughts. There will not be enough time for the memory to become intrusive. With these assurances, clients are usually willing to access memories that they might otherwise have avoided. After 6-12 appropriately brief flash passes, clients usually report a significant reduction in the disturbance associated with the memory, and clients are ready to proceed with the assessment phase of EMDR and the rest of the standard protocol.

\section{Description of Four Cases}

Four cases are presented here briefly, in which FT was used by four different therapists in the preparation phase of EMDR treatment. The targets in all four cases had a reported SUD level of 9 or 10. In each case, the target was extremely disturbing, and the therapist anticipated that processing would be challenging, either because clients had a tendency to shut down rather than process (Cases 1 and 2) or clients stated that they were unwilling to think about the target (Cases 3 and 4). FT was expected to minimize the client's defenses and make the processing rapid with no significant disturbance for the client. In all but case 2, the case with the child client, the standard EMDR protocol was used after the SUD was reduced to a moderate level. The results in all four cases remained stable through follow-up.

\section{Description of Case 1}

Client Presentation. Martin was an intelligent, educated, 35-year-old male who sought treatment a year after a rear-end collision that damaged his spine and 
resulted in multiple surgeries. Although his medical condition remained unresolved at the time he sought psychotherapy, he thought that getting treatment to help him deal with the trauma of the accident might make his current life less distressing. He described his life as a mess, saying he was not sleeping or eating, had flashbacks whenever he got into a car, and had not been able to hold down a full-time job since the accident.

Dissociative Features. Martin described that he had been moderately dissociative from an early age, at times becoming disoriented, and not knowing where he was or the reason for being there. He also reported at times being unable to feel physical pain. The precise diagnosis of his dissociative symptoms was unclear. He met the diagnostic criteria for PTSD. He rated the accident as 12 on the SUD scale of $0-10$. Nevertheless, he did seem well-resourced, as evidenced by an ability to think of a rich collection of positive memories.

Martin's PTSD dissociative patterns started long before the accident. However, Martin reported feeling fairly present in the treatment room as long as he was not reminded of the accident. Small reminders of the accident, however, had often resulted in reliving the accident and flooding. The most disturbing part of the accident for him was the moment of impact.

Decision to Use Flash Technique. Martin would certainly have benefited from ongoing treatment involving resourcing, psychoeducation, and working with dissociated parts. He did not view himself, however, as signing up for a long therapeutic campaign. The therapist (PM) wanted to provide the immediate relief Martin was specifically requesting.

The therapist anticipated that proceeding to the assessment phase of EMDR therapy would involve thinking of the accident and would probably result in Martin's reliving it and then shutting down, which was how Martin typically protected himself. Rather than engaging in an elaborate process of managing dissociation, resourcing the client, and titrating the disturbance associated with the memory, it was decided to use FT in preparation for the EMDR standard protocol to attempt to achieve some rapid reduction of the immediate high level of disturbance.

Twenty Minutes of Flash Technique. No assessment phase was attempted before using FT. After some stabilization exercises, 20 minutes of the session remained and FT was begun. The client was asked to focus on being safe and comfortable, then to flash extremely briefly on the memory of the accident impact, and then to come back to the comfortable state. The therapist emphasized the importance of making the flash so rapid that there would not be time for the memory to become disturbing. Nor would there be time to recall any action sequences from the memory or even to think about the memory.

The first flash set was the only one for which Martin reported any difficulty coming back to a neutral state, although he was able to. After that, he was consistently able to easily return to the calm state. By the fourth set, he reported that the memory seemed far away and that he was having difficulty accessing it. By the 14th set, the experience of the accident impact was difficult for him to find in his memory, and it appeared that he was ready to process the full memory because his focus shifted to the frightening experience of seeing the other car coming up in his rear view mirror before the impact. Time ran out in the session, and it was agreed that this other aspect of the memory would be processed in the next session. There was an evenness to Martin's disposition during the processing that continued through the end of the session. Before leaving, he reported feeling stable with a sense of well-being.

Subsequent EMDR Processing Without Flash Technique. In the following session, 2 weeks later, Martin reported that now he had no reaction to thinking about the crash, the subject of his flashbacks for the past year. He was having very little difficulty riding in a car, but he was still triggered by seeing movement in the rearview mirror. Although this aspect of the accident memory was reported as having an SUD level of 9 , it was characterized as less disturbing than the memory of the impact had been, and the client did not seem resistant to processing it directly with the standard EMDR protocol without FT. The result was a reported SUD level of 0 at the end of the session

Flash Technique Required. At reevaluation a week later, however, the SUD level associated with the rearview mirror image was reported as 6 . The therapist conceptualized that Martin's reported SUD level of 0 in the previous session had been the result of dissociation to avoid the intensity of the disturbance and that even Martin's current reported SUD of 6 would have been much higher if he had allowed himself to "go there." FT was used again to enable him to tolerate the disturbance without avoiding it. With FT, the disturbance was bought down to a true 6 , and the rest of the EMDR protocol was then used successfully to reduce the SUD level to 0 . The session ended with the client feeling full resolution about the memory.

Follow-Up. The next week, Martin still had no difficulty thinking about any aspect of the accident, and the SUD rating was still a 0 . The therapist reminded 
him that the first time his rearview mirror memory was processed, the SUD level had gone down to 0 , but it had jumped back up to 6 in the following session. He asked if Martin thought there was any possibility that this could happen again. Martin thanked him for asking and said, "No, this time is different. I'm not becoming startled in the car or anything." These results held at 3-month follow-up.

\section{Description of Case 2}

Client History. Mason was a 6-year-old boy whose adoptive parents brought him to therapy for attachment work and EMDR. Before this, Mason had had play therapy for 2 years but continued to have problems with prolonged tantrums once or twice a week, aggressive behaviors, food hoarding, hypervigilance, bedwetting, low self-esteem, disturbed sleep, and an intense fear of the dark that made bedtime very difficult. These symptoms were consistent with a history of complex developmental trauma and a diagnosis of PTSD and attachment disorder.

Mason's intense fear of the dark seemed to stem from an experience, at one of his foster placements, of being locked in a dark room by himself at night. He remembered being terrified of being alone in the dark and being unable to leave the room to go to the bathroom, so he wet himself at night. Now, despite adoption by a safe and loving family, he was disturbed by that memory.

Level of Disturbance. The therapist (JL) asked Mason to indicate how upsetting it was to remember being locked in the dark room by himself, using the distance between his outstretched hands as an SUD rating, with hands together indicating calm and neutral and hands $10 \mathrm{in}$. apart indicating maximum distress (SUD level $=10$ ). Mason flung his arms out as wide as they could go to indicate his overwhelming distress. Because his SUD rating was so high, and because he had been so traumatized from an early age, the therapist was concerned that Mason would feel overwhelmed if she tried to do EMDR processing with him without initial resource development and use of FT.

Establishing Present Safety. The therapist began with resource building and steps to establish present safety. She asked Mason's father if Mason would ever be locked in a dark room at their home and, of course, his dad reassured him that this would never happen. She added, "So, you can relax at night and know you will be safe." The therapist suggested that Mason's dad put his arm around Mason to reassure him that he is safe now. She asked Mason to feel his feet on the floor and his dad's arm around his shoulders, and said, "You and your dad and I are in my room now, and you are safe, and everything is OK. Can you feel that?" Mason nodded.

Implementing Flash Technique. The therapist asked Mason to "take a quick peek" at his distress at being closed in the dark room at the foster home and then to come back quickly to being in her room with his dad and her. She told him to take a peek so fast that he would not have time to feel anything or think anything and then to come back and say, "I'm back," or "Flash." While he "took a peek," she tapped his knees, and when he said, "I'm back," she stopped. Then she asked him to feel his dad holding him and feel his feet on the floor. When he nodded, she continued, "Are you ready to take a peek?" When he nodded again, the sequence was repeated, continuing the alternation between feeling present and safe and "taking a peek."

Results. Within 10 minutes, Mason's SUD level dropped from 10 to 0 , and he said the memory did not bother him anymore. Extremely rapid processing is not uncommon for children, but this session stood out because, during the flashing, Mason did not appear to be experiencing any noticeable disturbance. The rest of the EMDR standard protocol was skipped because there seemed to be no further disturbing images, feelings, or physical sensations. The SUD level was 0 and the body scan was clear. The intention had been to follow with the full EMDR protocol for desensitization and reprocessing, but that was unnecessary. Not having to access emotion and physical sensations was a clear advantage in working with this child who had such an extensive trauma background.

Follow-Up. At 1, 2, and 3 weeks' follow-up, Mason's parents reported that bedtime was much easier and that Mason was no longer afraid of the dark, which he verified. He also slept an additional hour per night, possibly indicating increased relaxation. These results continued to hold at 6 months.

\section{Description of Case 3}

Client Presentation. Jenna was a 34-year-old female, human resources director at a small tech company. Her older sister had been brutally murdered by a jealous, drugged-up boyfriend 3 years before the present series of consultations. Jenna had been depressed, irritable, and preoccupied in a way that impaired her job performance and was also threatening her marriage.

Initial Treatment. After taking a history, getting a list of her top 10 worst experiences, establishing a safe person and container, and teaching her some marital 
communication skills in her first two sessions, in Session 3, the therapist (LE) initiated processing of traumatic memories. A trauma memory that started at an SUD level of 6 was successfully processed using the standard EMDR protocol.

Overwhelming Disturbance. The second memory, her sister's murder, was first rated at an SUD level of 7 but was obviously much higher. It took two sessions of standard EMDR to achieve complete resolution. However, she avoided focusing on the most painful part of that set of events, which was identifying her sister's battered body in the town morgue. This last part of the experience was so overwhelmingly painful that she suffered daily from intrusive memories of it and she said that she just did not want to bring it to mind, which, of course, would have been necessary for standard EMDR. She clearly met the criteria for PTSD.

Flash Technique Processing. Because she was told that she could do FT without needing to recall the memory vividly, she agreed to try it. She rated her SUD level at 9. After the first three sets of FT, she reported no change and reported some difficulty returning from the flash exposures. She was instructed to make contact with the memory significantly briefer. By the fifth set, she was able to access the memory during the split-second flash without seeing details or hearing a dialogue.

Subsequent flash sets were barely disturbing if at all. After the ninth flash set, the SUD level was at 2. After four more sets, she could see the image without feeling disturbance. "It still looks terrible, but I'm just not having the pain." Other channels of that target were still unresolved, including one with an SUD rating of 7 , and standard EMDR assessment, desensitization, and installation phases allowed the processing to be completed. SUD score was then reported as 0 , and there appeared to be no disturbance at all at the end of the 45-minute session. The body scan was clear.

Follow-Up. At 10-day follow-up, the client reported that her reactivity to the memory was completely gone. These results continued to hold at 6-month follow-up.

\section{Description of Case 4}

Client Presentation. Charles was a 48-year-old adult male who presented with hypervigilance, nightmares, and avoidance behaviors. He also described himself as depressed for much of his adult life. The primary diagnosis was PTSD stemming from a driveby shooting in his early 20 s. For reasons that became apparent later, the client was unwilling to describe the details of this incident with the therapist (DM). He later shared, during processing, that one of his peers had been shot and killed. Charles had been strafed by automatic gunfire and recalled running for his life. Since the incident, he reported routine "checking over [his] shoulder," "scanning the room," and assuring himself he was familiar with all exits at all times.

Choice to Use Flash Technique. Charles agreed that this incident was undoubtedly the source of his presenting symptoms, most notably flashbacks, avoidance, and hypervigilance. Nonetheless, he was ambivalent about EMDR, and he was unwilling to revisit the memory at all. After successfully using standard EMDR procedures to target some less threatening material in our second session, it was suggested to Charles that a novel approach to EMDR might allow him to approach the drive-by memory without overwhelming him with detail. Charles agreed to a third session but shared later that he almost did not show.

Implementation of Flash Technique. The client was not asked to identify the traumatic moment, nor create a target image. No assessment phase was done. In an oblique reference to the memory, he was simply asked to "flash on that on the count of three." Typical of FT, as the SUD level began to decline, there was no overt abreaction or shift in apparent affect. In other words, there was no visible indication that anything was changing. Initially, the client reported an SUD score of 12 on the 0 to 10 scale. After the first flash and accompanying set of EM passed, he reported little change. However, the second set resulted in a comment about the memory being slightly less disturbing, and several more sets reduced the disturbance to a 6 .

At this point, Charles was willing to share the details of his memory, and processing proceeded using standard EMDR procedures without FT. Subsequent sets focused on pools of blood, sounds of bullets, and the death of his friend. Processing current and potential future events evoking an irrational perception of danger helped secure his sense of safety.

Follow-Up. Reevaluation the following week found no disturbance associated with this memory. Charles felt more relaxed with a full resolution of his fear and hyperarousal. These results held at 3-month follow-up.

\section{Discussion}

\section{Summary}

Four case examples were described involving clients with PTSD from overwhelmingly disturbing 
events. In each case, it was evident that the client was consciously or unconsciously avoiding accessing the disturbance, making the use of FT ideal, because it bypasses client resistance. In Case 1, after processing the most disturbing aspect of a car accident with a moderately dissociative client using FT, EMDR without FT was used to process the second most disturbing aspect of the accident, and the processing was not successful. In the following session, FT was used successfully with that aspect of the memory, followed by standard EMDR procedure. In all four cases, FT was used effectively to rapidly reduce the clients' associated disturbance levels, and the clients experienced little disturbance during FT processing (see Table 1).

\section{Proposed Mechanism of Action}

Multiple Proposed Mechanisms. Most clients with PTSD reexperience aspects of their traumatic memories when they think of them (Hackmann, Ehlers, Speckens, \& Clark, 2004; R. A. Lanius et al., 2010; Shapiro, 2001). We suggest that one reason for the effectiveness of FT is that it prevents reexperiencing of traumatic memories by radically reducing the amount of time available to access the memory. In addition, FT does not permit the client enough time to bring up a clear memory of the target or to think about it. We believe that this aspect of FT interrupts conscious defenses against accessing the memory. Also, the repeated alternation between the neutral, present state, and accessing the past memory with relatively little emotion emphasizes that "that was then and this is now." Although we cannot rule out the possibility that the effects of FT can be explained by WM, in our opinion, WM cannot account for the effects. Given the rapidity of effects, we strongly believe that FT has a unique mechanism of action.

Ecker et al. (2012) and Lee (2009) proposed that for effective memory reconsolidation, there must be a contradictory experience. The FT appears to produce several possible contradictory experiences, most prominently

- Decreased affect and emotion because of working memory effects as central executive resources are required for accessing/dismissing memory, with simultaneous EM (Gunter \& Bodner, 2008; Shapiro, 2001)

- Relaxation effects because of physiological effects of EM (Kreyer \& Egon, 2008; Schubert et al., 2011)

- Sense of efficacy because of ability to repeatedly access and dismiss the memory

- Observer position and mindful stance, providing a sense of distance and acceptance
- Inhibition of conscious defense mechanisms, preventing potential dissociation, avoidance, or abreaction

- Consolidation of past-present perspective because of frequent brief shifts of attention

Adaptive Information Processing. Consistent with the adaptive information processing (AIP) model of EMDR trauma resolution, as the client repeatedly takes more of an observer position toward the trauma memory, the client's adaptive adult perspective begins to come to awareness. Applying Shapiro's (2001) model, we hypothesize that FT procedure may lay down new neural pathways between the trauma memory network and memory networks linked to these new perspectives and that this might facilitate healing and perhaps some correction of cognitive distortions.

\section{Differences Between Flash Technique and Other Forms of Titration}

Unlike most other forms of titration, FT helps the client to focus on the undiluted, disturbing memory, but for a very limited time. The time of contact with the memory is what is titrated. The amount of time allowed in FT is many times shorter than in other forms of titration. In contrast to other forms of titration, FT is extremely rapid. Also, with FT, clients appear to be almost disconnected from the memory emotionally, and it is sometimes hard to tell from observation alone if any change has occurred.

\section{Limitations of Flash Technique}

Although FT appears to reduce disturbance substantially, it usually does not fully process memories or bring their disturbance down to 0 . The client does not have the benefit of having done an assessment to clarify the components of the target. Also, if there are multiple channels contributing to the client's disturbance, FT, as currently practiced, often does not bring secondary channels into focus or have an impact on them. In FT processing, cognitions are not discussed. Because there has been no assessment phase before the use of FT, cognitive distortions have not been initially identified and often are not fully clarified in the processing, even though some adaptive adult perspectives usually emerge. Clients who are highly dissociative or otherwise unable to feel reasonably calm and safe, even before focusing on the disturbing material must be helped to connect to a calm state. If they cannot, they are not good candidates for FT. Also, FT is 
unlikely to be fully effective if the target memory has a feeder memory that is not being addressed.

\section{No Reported Adverse Client Responses}

To date, the results of approximately 200 sessions using this technique have been informally collected. No adverse client responses have been reported, other than a few abreactions that responded easily to a standard set of rapid BLS with cadence comments.

\section{Indications for the Use of Flash Technique}

It should be highlighted that FT is recommended for use in EMDR's preparation phase, or to address blocked processing in the desensitization phase. It is not presented as a substitute for full EMDR treatment. This technique seems to be most useful for clients who are not highly dissociative but resist fully accessing intense disturbance. Although a highly skilled EMDR clinician might be able to coax such a client eventually to access a very disturbing memory fully, FT accomplishes this in a relatively effortless and painless way and clients seem to become less fearful about becoming overwhelmed.

\section{Ease of Learning}

The technique seems to be relatively easy for EMDR clinicians to learn. Of the approximately 20 EMDRtrained clinicians who have been given at least 20 minutes of instruction and been provided with three short video demonstrations of the technique, all have reported achieving some positive results with FT. More detailed training improves results.

\section{Recommendations for Further Study}

A study comparing the effectiveness on two groups of matched clients with extremely disturbing memories, one receiving standard EMDR treatment and the other EMDR with FT included in the preparation phase, would help to establish how much of a difference, if any, this technique makes.

A study designed to determine what duration of flash is optimal could be very useful. It is possible that preparing clients for FT by showing them a short video illustrating different lengths of flashes and letting them know what is optimal would further reduce the learning curve for clients who initially make their flashes too long. Alternatively, teaching FT by beginning with a less disturbing target, like a disturbing scene from a movie, may enable clients to learn to flash with minimal exposure to the memory. A study to test whether FT becomes easier and quicker for clients after they have initially learned how to do flash briefly enough on their memories and whether this accelerates or diminishes the effectiveness of FT would presumably help in evaluating the working memory hypothesis as it relates to FT.

Additional Questions. Does FT require EM? It would be valuable to conduct research comparing FT with EM to FT without EM/BLS. It would also be valuable to compare FT with EM to FT with bilateral tapping and/or auditory stimulation. In addition, research evaluating each of the mechanisms of action proposed in this article would help in further understanding FT.

\section{Conclusion}

This article presented four treatment accounts that used FT, a method of rapidly reducing disturbance levels in preparation for EMDR trauma processing. The technique appears to be effective, rapid, easily tolerated, and easily taught to practitioners. To date, we have identified no significant risk factors associated with its use. More study is warranted.

\section{References}

Barreiro, K. A., Suárez, L. D., Lynch, V. M., Molina, V. A., \& Delorenzi, A. (2013). Memory expression is independent of memory labilization/reconsolidation. Neurobiology of Learning and Memory, 106, 283-291.

Dudai, Y. (2004). The neurobiology of consolidations, or, how stable is the engram? Annual Review of Psychology, 55, 51-86.

Ecker, B., Ticic, R., \& Hulley, L. (2012). Unlocking the emotional brain: Eliminating symptoms at their roots using memory reconsolidation. New York, NY: Routledge.

Elsey, J. W., \& Kindt, M. (2017). Tackling maladaptive memories through reconsolidation: From neural to clinical science. Neurobiology of Learning and Memory, 142, 108-117.

Forgash, C. (2004, June). Healing the heart of trauma: Restoring connections and stability. Paper presented at the 5th EMDR Europe Association Conference, Stockholm, Sweden.

Gomez, A. (2014, September). There and back again: The intricate and fascinating journey of helping children with complex trauma using EMDR therapy. Paper presented at the 20th EMDR International Association Conference, Denver, CO.

Greenwald, R. (2008). Progressive counting for trauma resolution: Three case studies. Traumatology, 14(4), 83-92.

Gunter, R. W., \& Bodner, G. E. (2008). How eye movements affect unpleasant memories: Support for a workingmemory account. Behaviour Research and Therapy, 46, 913-931. 
Hackmann, A., Ehlers, A., Speckens, A., \& Clark, D. M. (2004). Characteristics and content of intrusive memories in PTSD and their changes with treatment. Journal of Traumatic Stress, 17(3), 231-240. http://dx.doi .org/10.1023/b:jots.0000029266.88369.fd

Hupbach, A., Gomez, R., Hardt, O., \& Nadel, L. (2007). Reconsolidation of episodic memories: A subtle reminder triggers integration of new information. Learning \& Memory, 14(1-2), 47-53.

Kinowski, K. (2003). Put your best foot forward: An EMDRrelated protocol for empowerment using somatosensory and visual priming of resource experiences. Retrieved from http: / / www.krystynakinowski.com/manual.html

Knipe, J. (2008, June). EMDR toolbox. Paper presented at the 9th EMDR Europe Association Conference, London, United Kingdom.

Korn, D. L., \& Leeds, A. M. (2002). Preliminary evidence of efficacy for EMDR resource development and installation in the stabilization phase of treatment of complex posttraumatic stress disorder. Journal of Clinical Psychology, 58(12), 1465-1487. http://dx.doi .org/10.1002/jclp.10099

Kreyer, A. K., \& Egon, S. (2008, June). Physiological effects of eye movements of different speeds and eye fixation during engagement in negative autobiographical memories: Experimental research regarding EMDR. Paper presented at the 9th EMDR Europe Association Conference, London, United Kingdom.

Lanius, R. A., Vermetten, E., Loewenstein, R. J., Brand, B., Schmahl, C., Bremner, J. D., \& Spiegel, D. (2010). Emotion modulation in PTSD: Clinical and neurobiological evidence for a dissociative subtype. The American Journal of Psychiatry, 167(6), 640-647. http://dx.doi .org/10.1176/appi.ajp.2009.09081168

Lanius, U. (2005, April). 'Dissociative processes' and EMDRstaying connected. Paper presented at 3rd Annual Conference of the EMDR Association UK and Ireland, Jordanstown, Northern Ireland.

Lee, J. L. (2009). Reconsolidation: Maintaining memory relevance. Trends in Neurosciences, 32(8), 413-420.

Leutner, S., \& Cronauer, E. (2010). Stabilizing while processing -integration of resources into the EMDR protocol (RIT). Paper presented at the 11th EMDR Europe Association Conference, Hamburg, Germany.

Levine, P. (1997). Waking the tiger-healing trauma. Berkeley, CA: North Atlantic Books.

Manfield, D. C. (1986). Computer-assisted weight-loss: A subliminal and behavioral methodology for motivated females (Unpublished doctoral dissertation). University of Wisconsin, Madison, WI.

Manfield, P. (2010). Dyadic resourcing: Creating a foundation for processing trauma. Berkeley, CA: Cornucopia.

Manfield, P. (2013). EMDR up close: Subtleties of trauma processing. Berkeley, CA: Cornucopia.
Manfield, P., \& Shapiro, F. (2003). The application of EMDR to the treatment of personality disorders. In J. F. Magnavita (Ed.), Handbook of personality disorders: Theory and practice (pp. 304-330). New York, NY: Wiley.

Mansfield, D. J. (1997). Subliminal priming and mood: A preliminary study (master's thesis). University of Natal, Peitermaritzburg, South Africa.

Maxfield, L., Melnyk, W. T., \& Hayman, G. C. (2008). A working memory explanation for the effects of eye movements in EMDR. Journal of EMDR Practice and Research, 2(4), 247-261. http: / / dx.doi.org/10.1891/1933-3196.2.4.247

Meyer, C., \& Waller, G. (1999). The impact of emotion upon eating behavior: The role of subliminal visual processing of threat cues. International Journal of Eating Disorders, 25(3), 319-326. http://dx.doi.org/10.1002/ (sici)1098-108x(199904)25:33.0.co;2-9

Ochberg, F. (1996). The counting method for ameliorating traumatic memories. Journal of Traumatic Stress, 9, 873-880

Pedreira, M. E., Pérez-Cuesta, L. M., \& Maldonado, H. (2004). Mismatch between what is expected and what actually occurs triggers memory reconsolidation or extinction. Learning \& Memory, 11(5), 579-585.

Schubert, S. J., Lee, C. W., \& Drummond, P. D. (2011). The efficacy and psychophysiological correlates of dualattention tasks in eye movement desensitization and reprocessing (EMDR). Journal of Anxiety Disorders, 25(1), $1-11$.

Sevenster, D., Beckers, T., \& Kindt, M. (2013). Prediction error governs pharmacologically induced amnesia for learned fear. Science, 339(6121), 830-833.

Shapiro, F. (1989). Efficacy of the eye movement desensitization procedure in the treatment of traumatic memories. Journal of Traumatic Stress, 2(2), 199-223.

Shapiro, F. (2001). Eye movement desensitization and reprocessing (EMDR): Basic principles, protocols, and procedures (2nd ed.). New York, NY: Guilford Press.

Silverman, L. H., \& Weinberger, J. (1985). Mommy and I are one: Implications for psychotherapy. American Psychologist, 40(12), 1296-1308. http://dx.doi .org/10.1037/0003-066x.40.12.1296

van Veen, S. C., van Schie, K., Wijngaards-de Meij, L. D., Littel, M., Engelhard, I. M., \& van den Hout, M. A. (2015). Speed matters: Relationship between speed of eye movements and modification of aversive autobiographical memories. Frontiers in Psychiatry, 6, 45. http:/ / dx.doi.org/ 10.3389/fpsyt.2015.00045

Wolpe, J. (1958). Psychotherapy by reciprocal inhibition. Stanford, CA: Stanford University Press.

Correspondence regarding this article should be directed to Philip Manfield, PhD, John F. Kennedy University, 2936 Domingo Ave., Suite 4, Berkeley, CA 94705. E-mail: philip .manfield@gmail.com 